\title{
Which way is upright and normal? Haptic perception of letters above head level
}

\author{
GIUSEPPE MIRABELLA and JOHN M. KENNEDY \\ University of Toronto, Toronto, Ontario, Canada
}

\begin{abstract}
In three experiments, the perception of the apparent orientation of block letters shown in various orientations above the subject's head in the horizontal plane was examined. A block letter $F$ with its front facing down toward the observer has two crossbars on its right side; the top is the part with the long crossbar, and the base has no bar. The experiments involved changing the locations of these parts with respect to the observer. In Experiment 1, the subjects using touch most often identified a letter as having its left and right sides in a normal orientation if the front of the block letter faced upwards away from the observer, with the bar on the right and the top of the letter farther from the subject than the letter's base. In Experiment 2, the subjects judging visual uprightness favored positions in which the bars were on the right, the top of the block letter was near them, and the letter's front faced downwards toward the observer. In Experiment 3, the subjects using touch most often assessed letters as being upright if the top of the letter was the farthest part and the bar was on the right. The results suggest that, when assessing orientation, subjects using touch favored positions that would be reached by a letter moving vertically upwards from table height, but subjects relying on vision favored positions reached by a letter moving in an arc centered on the subject's head (on the eyes, in particular).
\end{abstract}

Both vision and touch are concerned with spatial issues. Indeed, although vision and touch are not identical in their reactions to spatial matters, many assessments of shape made in vision and in touch are the same or tackle similar issues. Novel shape perception and categorization tasks (Garbin, 1988), letter-recognition tasks and subsequent assessments of apparent orientation (Marmor \& Zaback, 1976; Oldfield \& Phillips, 1983), drawingrecognition tasks (Kennedy, 1993, 1997), even some illusions, such as the Bourdon (Day, 1990) and MuellerLyer (Rudel \& Teuber, 1964) illusions, all have many similar effects when presented in the visual and tactile modalities. Also, practice effects in one modality can be transferred to another (Rudel \& Teuber, 1964). Results such as these suggest that, in some respects, haptic and visual modalities provide the observer with spatial information in much the same way (Garbin, 1988; Garbin \& Bernstein, 1984; Jones, 1981; Marks, 1978). If so, threedimensional (3-D) space may be as relevant to touch as to vision (Kennedy, 1993; Kennedy, Gabias, \& Heller, 1992).

However, to say that the problems being tackled by the two senses are the same is not to claim that vision and

This study is based on the first author's Master's thesis. Thanks are due to Patrick Cabe, Gregory Burton, Lester Krueger, and an anonymous reviewer for commentary during the preparation and revision of this article. We especially thank Allison Sekuler for her suggestions and assistance. Thanks also go to Julie Seemangul and Kavita Seeratan for assistance in running subjects. Correspondence concerning this article should be sent to J. M. Kennedy, University of Toronto, 1265 Military Trail, Scarborough, ON M1C 1A4, Canada (e-mail: kennedy@lake. scar.utoronto.ca).

-Accepted by previous editor, Myron L. Braunstein touch come to identical conclusions about spatial matters. Indeed, here we will report on some judgments in which observers assessing the apparent orientation of letters with touch react to the letter's orientation in a way vision ordinarily does not.

Often, vision and touch respond to spatial information in the same way so that we can function efficiently in the everyday world. For example, the observer, seated at a table or desk, can reach out to pick up objects in a space that includes the tabletop, drawers under it, the floor, and shelves at various heights above the tabletop. An object can be located at any of these heights and can be to the left, the right, or straight ahead. The observer often needs to pick up an object, such as a jar, at one elevation and move it to another, while keeping its orientation constant. Drivers of cars and pilots of airplanes reach up for light switches, to one side for objects in glove compartments, and down for cups full of liquid. Touch must provide information about the location of the target objects and their orientation in space. Is the cup of hot liquid upright or tilted? Is the switch on or off, up or down? Indicators, letters, signs, handles, detached objects, and containers have 3-D locations, and perception - including touch-must provide assessments of their orientations before and after changes in these locations.

The present study is concerned with the tactile perception of objects in locations in 3-D space near to and in front of the observer. In particular, the assessment by subjects through touch of the apparent orientation of a letter when it is placed in various physical orientations above head level in the horizontal plane is investigated. Furthermore, assessments of letters, made through touch, that are different from assessments that are made through vision 


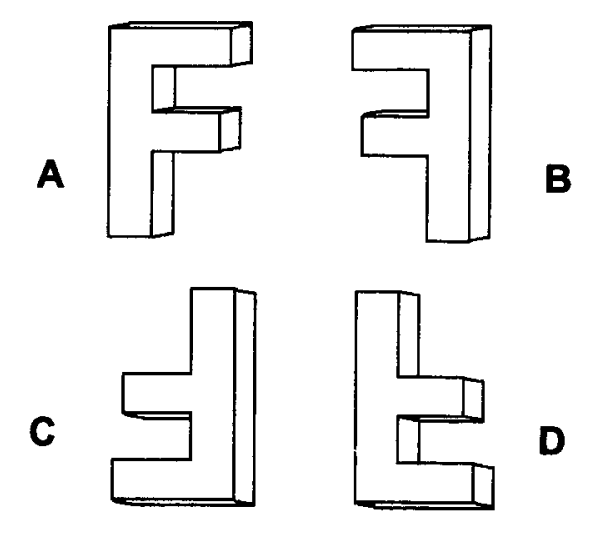

Figure 1. The letter $F$ in one of four postures: (A) normal, upright, (B) left-right mirror reversed, (C) upside down, and (D) upside down and left-right reversed.

are investigated. We will argue that touch may be described as predominantly relying on positions that would be reached by using a different spatial transformation than that which vision ordinarily applies to letters.

\section{Spatial Transformations}

Before defining how the apparent orientation of a letter might be perceived through touch, it is first necessary to describe some ways a letter's orientation is perceived in vision. We will discuss orientations of a letter on a table and ones that are achieved by moving the letter from the table, in what we will term motion in an arc around a fixed point and motion that consists of a change of elevation. We will note that these spatial transformations affect what parts of an object are near to the observer and what parts are far from the observer. We will also consider the effects of changing which parts of an object are on the subject's left and right. These changes indicate where the front of an object is facing, and they have implications for the observer's vantage point.

Arc transformation. Consider the words printed on this page. If you are reading this page resting flat on a table, it is likely that every letter is positioned in its upright, normal position, relative to you: The top of each letter is the part that is furthest from you. Also, the letter might be said to show its front to you; that is, if the page were held up to a strong light and the reverse side of the page were turned toward you, so that this sentence would be viewed through the page, you would be looking at the back of the letter. If we made a letter out of wood and placed it in the same position as that of the letters on this page, we would distinguish the front, back, top, and bottom of a letter such as an F more readily. In Figure 1A, the front of a sans serif block letter $F$ has a vertical straight stem on the observer's left and two straight horizontal pieces branching out from the top and the midsection of the letter.

In Figure 1, panel A shows the front of a block letter $F$, and panel B shows the letter turned over to face away from the observer and, thus, reveal its back. Panel $\mathrm{C}$ also shows the front of the letter, since it shows A turned $180^{\circ}$ in the plane. Panel D shows the back of the letter, since it shows B turned $180^{\circ}$ in the plane. When Figure 1 is set horizontally on a table, panels $A$ and $C$ show a letter facing upwards toward the observer, and panels $B$ and D show a letter facing downwards away from the observer. If the figure is held above one's head so that its caption can still be read, $A$ and $C$ would face downwards toward the observer, and B and D upwards away from the observer. To make some of the observations discussed here, this figure can be viewed in several positions: first, while the figure is lying on the table; second, while it is held vertically as if attached to a wall; and third, while held above the reader's head and slightly in front of the reader, as if attached to the ceiling. In the first position, the viewer is looking down; in the second position, the viewer is looking horizontally; and in the third position, the viewer is looking upwards. These three positions are on an arc centered on the observer's head.

Now, if you were simply asked to attach this page to a wall in front of you, so that you could read it, you would probably place it in such a way that, from your perspective, every letter would have its front facing directly toward you and its top would be the part that is farthest from the floor. The top and bottom would be equidistant from your vantage point.

What if you were asked to attach this piece of paper to the ceiling and slightly in front of you, so that you could still read it with a modest tilt of your head? Here, you would position it so that, from your vantage point, the front of the letter would be facing down to the floor and toward you. Notice that the top of the letter would be the part that is nearest to you. When the letter was on the table, the top was the part that was farthest from you.

The letters described here as being placed on the table, on the wall, and on the ceiling in the readable position always appear upright to you. These positions are illustrated in Figure 2. Interestingly, they appear upright despite several positional changes relative to you and in particular, they appear upright despite the fact that the top of the letter is sometimes the furthest part from you and sometimes the nearest part. Furthermore, if you follow the path of the block letter $F$ and the letters on the page as they move from the table, to the wall, to the ceiling, you will note that the letters travel in an arc centered on your head (on your eyes, in particular). At every point along the arc, the letters are seen as being upright and normal-the standard and ordinary position of the letter. To be upright, our considerations reveal, the top of the letter should be above the base of the letter in a direction from the observer's vantage point. An arc transformation preserves this relation between the direction of the top and the direction of the base from the observer's vantage point.

Of course, a letter is not always observed in its upright, normal position. There are two major ways in which to deviate from the normal position: The letter may be presented with its left and right sides reversed or upside down. In Figure 1, position B is left-right mirror reversed, $\mathrm{C}$ is upside down through being rotated $180^{\circ}$ in the plane, and $\mathrm{D}$ is left-right mirror reversed and upside down. In- 

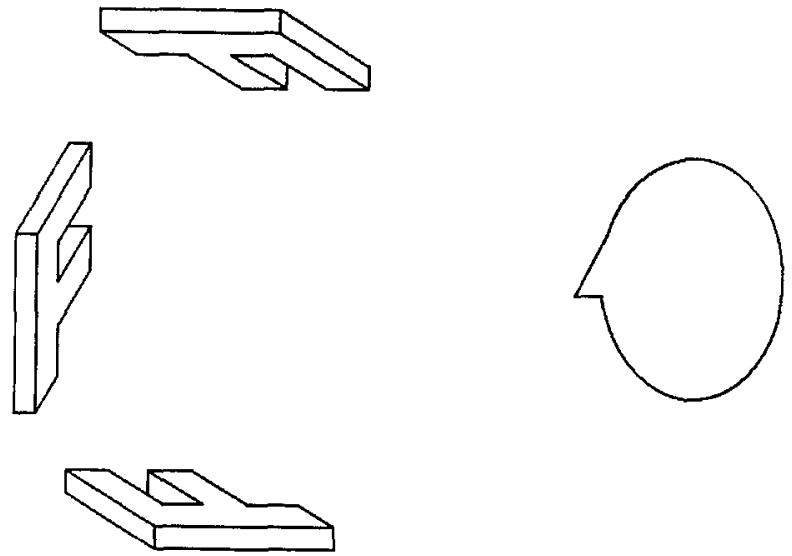

Figure 2. The are transformation from a horizontal position below to one above head level.

structively, if a letter on the table is not in its normal orientation, through being left-right reversed or inverted, it appears to maintain this nonstandard orientation if it travels along an arc centered on your head from the table, to the wall, to the ceiling. The reader can test this claim, using Figure 1, by monitoring the apparent orientations of $\mathrm{B}, \mathrm{C}$, and $\mathrm{D}$ with an arc transformation. The reader will find that the arc transformation does not disturb the apparent orientation of the letter forms, whether the letter is in a nonstandard position or not.

Elevator transformation. Is an arc transformation the only transformation that can maintain an object's apparent orientation as it moves in 3-D space in front of the observer? No, it is not. Consider a cup of liquid, for example. Let the cup be put on a table, with its handle nearest to you. If the cup is raised to the ceiling following an arc centered on your head, the result will be that the cup will spill the liquid that was in it. That is, an arc transformation changes the apparent orientation of the cup from a posture in which it can contain liquid to one in which it cannot. However, there is a transformation that maintains the apparent orientation of the cup as it changes in height: It must be moved vertically upwards, as if on an elevator, as in Figure 3, in which an F is moved upwards while remaining horizontal in posture. The position of the cup's handle would remain in the same position with respect to you during an elevator raise; that is, the parts of the cup that are on the left and the right, or near or far, remain in those positions. The elevator transformation preserves the relative distances between the parts of the cup and the frontal plane of the observer. The part that is closest to the frontal plane remains closest.

The description given so far applies to vision. Is it applicable to touch? How will letters be perceived through touch if they are horizontal but above the observer's head? One possibility is that they would follow the same rules as those followed by the letters in our discussion above. That is, the arc transformation described above, centered on the observer's head, might leave the letter's tactile orientation invariant. A second possibility is that the vertical elevator raise, mentioned in connection with a cup, might leave a tactile letter's apparent orientation invariant. A third possibility is that there will be a mixture of these two systems in touch. That is, whereas vision may consistently rely on positions reached via the arc transformation for letters, touch may be inconsistent and use the arc and elevator transformations at different times.

There is reason to anticipate that, although subjects may assess the orientation of letters through vision as if they were using an arc transformation, subjects engaged in a similar task with touch might not use vision's rules. Touch is often used to elevate manipulable objects that must maintain their openings upwards to retain their affordances (Gibson, 1979) - that is, arrows, drawers, cups, pendulums, and so forth. The affordances of these objects are often defined with respect to gravity and not with respect to the observer's vantage point. Such an important transformation in touch may even serve as the default mode of assessing orientation. But a second factor in orientation is the object that is being perceived. Certain kinds of objects are inherently defined with respect to an observer. Letters, for example, have no other use than their cultural use, and a $p$ and a $d$ are distinguished by their orientation to the context and the observer, not by their shape. So, whereas in both vision and touch, containers function with respect to gravity, letters are defined in ways that fit with arc transformations.

In sum, there are two kinds of influences that can be pitted against one another. Letters are relatively unfamiliar objects in touch, so a transformation (the elevator) that is common in the use of touch because of touch's target objects might often be applied in a tactile task to an object that ordinarily belongs with a different transformation (the arc) in a different modality (vision).

Vantage point. The arc and elevator transformations leave the left and the right sides of letters invariant. Figure $1 \mathrm{~B}$ shows the effect of reversing left and right and
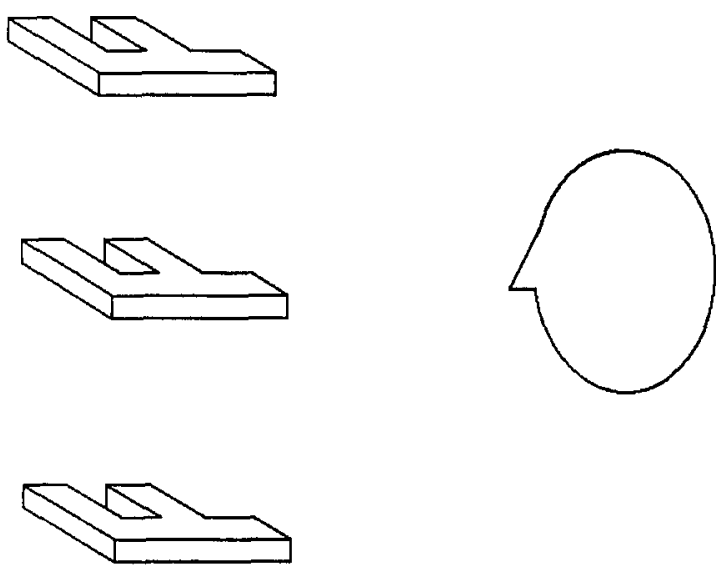

Figure 3. The elevator transformation from a horizontal position below to one above head level. 
suggests a letter face down. The crossbars of an F project to the right. Figure 1B shows what appears if we take a page of normal printing in a book with thin, partially transparent pages and turn the page, but then observe the printing through the page. It follows that, if the reader turns the page bearing Figure 1B over and views through the page by holding the page with a light source behind it, Figure 1B showing through the page will appear to be a normal $\mathrm{F}$, accompanied by a left-right mirror-reversed $\mathrm{B}$ (its caption). What might happen if, instead of viewing through the page, we reached around to feel a shape like Figure 1B from underneath the page? We might locate our apparent vantage point on the underside of the letter, to fit with the direction from which we are reaching. The result would be that the letter would appear to be a normal F, albeit one on the reverse side of the page.

Oldfield and Phillips (1983) asked subjects to feel a plastic letter attached to a flat surface (at table level). The flat surface bearing the letter could be set to be in front of the subject, as if the letter was resting on the top surface of a table, or it could be turned over, as if the letter was attached to the underneath surface of the top of a table. In half the conditions, the subject's hand was above the letter (feeling the letter with the hand palm down), whereas in other conditions, it was below the letter (palm up), feeling the letter from underneath. Thus, the observer's hand approached the letter from above or below. The letters were presented in their normal, upright orientation, relative to the subject, or left-right reversed from the normal, upright orientation. The effect of their left-right reversal was that the letters changed from facing up and toward the ceiling to facing down to the ground.

The result of Oldfield and Phillips' (1983) manipulation was that subjects reported a difference in the perceived letter that depended on the direction from which the subject's hand approached the stimulus. For instance, $a b$ pattern felt from above in the palm-down condition was perceived as being $a b$, but if the subject felt this pattern from below, in the palm-up condition, it was perceived as being a d. The orientation of the sensing hand to the physical surface being felt changed the apparent orientation of the physical pattern from facing up to facing down. However, if Oldfield and Phillips had instructed their subjects that they were feeling the underside of the letter, what was called a $\mathrm{d}$ facing down (toward their hand) might often have been called a $b$. That is, apparent vantage point may be independent, to some extent, of the direction from which the observer's hand approaches the object. Also, if Oldfield and Phillips had told the subject the identity of the letter, they could have then asked about the letter's apparent orientation. Subjects given a letter $p$ without being told its identity and asked about its orientation may first ask whether the letter is $p, b, d$, or q. The letter's apparent orientation depends on its identity. With this consideration in mind, in our tactile studies, we informed our students of the letter's identity and inquired about its orientation.
In cutaneous perception tasks, forms such as letters are drawn, with some blunt stylus, on various areas of the subject's body. The subject reports the letter or number and its orientation. Results from these tasks directly indicate that subjects identifying cutaneous patterns may entertain a variety of vantage points in three dimensions. For example, Natsoulas (1966) drew the letter b, d, p, or q on the skin of the temples of subjects, who were asked to identify the letter. Subjects mainly used one of two vantage points when identifying the letter. One vantage point had an internal locus. The subject behaved as if looking at the stimulus from within his or her head. That is, the subject identified as a $d$ what appears to be $a b$ on the subject's temple to the experimenter. The other vantage point had an external locus, like a disembodied eye (Concoran, 1977), and was often the vantage point of the experimenter. Although it is not known why a particular vantage point is used in a particular instance and the selection may be readily malleable by instructions, the use of various vantage points in touch is likely a "process shared by blind and sighted individuals" (Shimojo, Sasaki, Parsons, \& Torii, 1989, p. 149) and is not solely visual or, it seems, due to any kind of visual imagery that requires sight (see also Kennedy, 1993, 1997; Sekiyama, 1991).

\section{The Present Study}

Three experiments will be reported. In the first, subjects using touch will assess whether a letter presented above the subject's head and slightly in front of the subject has its left and right sides reversed from its typical or standard position or not. This will enable us to test whether positions reached by arc or elevator transformations appear to the subject to offer normal or left-right mirror orientations. Does more than one position leave orientation invariant? Or is one preferred? In the second experiment, we will empirically substantiate the claim that a letter placed above a subject's head, slightly in front of the observer, with its top near the subject and facing downwards toward him or her (see Figure 2), is indeed seen as being in an upright position. This will establish the claim that, in vision, subjects assess the apparent orientation of letters as if they were using an arc transformation. In the third experiment, subjects will assess through touch whether a letter placed above the head is upright or not. If subjects assessing apparent orientations through touch take as their standard the position that would be reached by using an arc transformation, the physical position at "ceiling" level that has the standard orientation will be the position (1) in which the top is near the subject and the letter faces down, and (2) in which the left and right sides of a letter on a table should be the left and right positions of the letter above the subject's head. If subjects take as their standard the orientation that would be reached by using an elevator transformation, the top of the letter should be far from the subject, the letter should face up, and left and right sides should remain as they would be on the table. Also, orientations reached by either transfor- 


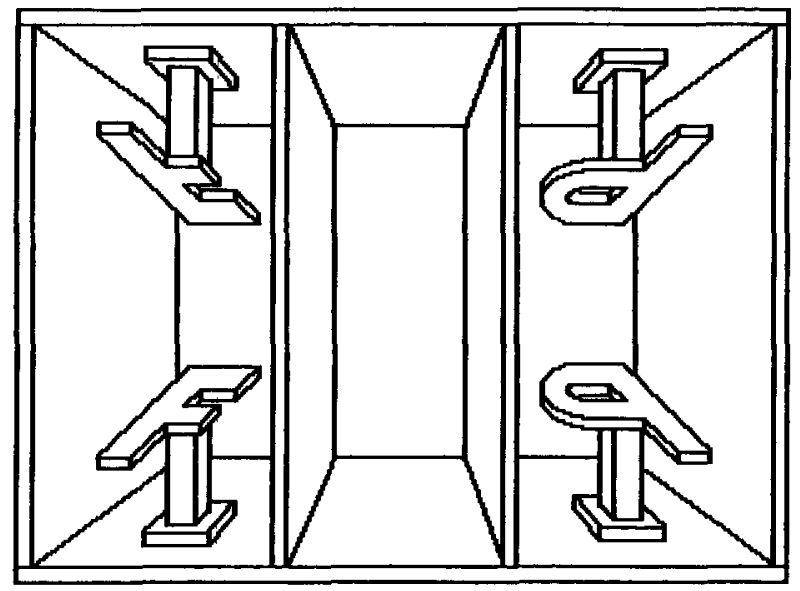

Figure 4. The mounting device with top:far letters attached. Top:near letters are not shown. In both the upper and the lower sections, the letter $F$ faces front:up, whereas the letter $P$ faces front:down.

mation may be equally available, or one may be favored but the other run a strong second.

Our stimuli were held on stems or columns and haptically explored. They were not presented as resting on a large flat surface. The letters were larger than the stem to which they were attached. The subject's fingers could wrap around the edges of the letters; that is, the letters could be grasped.

\section{EXPERIMENT 1}

Letters are objects that are relatively unfamiliar to touch. Does this mean that transformations such as the elevator that matter to touch could influence judgments of letter orientation? The first experiment on touch was concerned with the positions of parts of a letter on our left versus our right, with the letter being above the subject's head. The left-right positioning of the parts indicates which way the front of the letter faces. The letters were presented in several positions. One, in keeping with the arc transformation, had the top of the letter $F$ near to the subject and the crossbars of the letter on the subject's right. One, in keeping with the elevator transformation, had the top of the letter F far from the subject and the crossbars on the subject's right once again. There were two positions with the crossbars on the subject's left. One had the top of the letter far from the subject (and the letter facing downwards, toward vantage points below the letter), and one had the top near the subject (and the letter facing upwards, toward vantage points above the letter).

\section{Method}

\section{Subjects}

Twenty-five subjects ( 20 females, 5 males; mean age $=22.4$ years, range $=17-39$ ) participated in this experiment. Fifteen of these were volunteers from a third-year psychology course; the others participated for credit in an introductory psychology class.

\section{Stimuli and Apparatus}

Sans serif letters $F$ and $P$ were attached to a mounting device. The letters measured approximately $20 \mathrm{~cm}$ high $\times 14 \mathrm{~cm}$ wide $X$ $1.5 \mathrm{~cm}$ thick. Two models of each letter were used, so that all four positions (top:near or top:far and normal or left-right mirror reversed) could be presented to the subjects with minimal adjustment to the stimuli.

Each letter was attached to a tall, slim column or stand, $12 \times 4 \times$ $4 \mathrm{~cm}$, and so could be explored haptically from all sides, although the subjects might approach the letter from a given direction, above or below the letter. The letters on stands were mounted on a box-like device made of plywood (see Figure 4 ). The device provided an upper board $(120 \times 30.5 \mathrm{~cm})$, to which the stands could be bolted, to hold the stimuli at a level above the subject's head while they were seated. The upper board was supported on vertical boards which rested on a base, lower board (also $120 \times 30.5 \mathrm{~cm}$ ) sitting on the table. The upper board could hold three stimuli approximately $30 \mathrm{~cm}$ apart, separated by the vertical boards. A maximum of six stimuli could be attached to the device at any one time.

\section{Procedure}

The subjects were tested individually. The letters were presented in four orientations, relative to the observers. Each subject was given instructions and a practice session. The subjects were also told the identity of the test letters prior to testing, so that exploration time with the letter would be spent primarily assessing orientation.

Each subject was asked to assess the left-right positioning of the block letters $\mathrm{F}$ and $\mathrm{P}$, above their heads, in the horizontal plane. That is, they were to indicate whether the left and right sides of the letter were in their standard positions or whether they were reversed, regardless of the letter's upright positioning. If they were not reversed, they responded "normal"; if they were reversed, they responded "mirror."

Instructions. A sans serif block letter B (without an attached stand) was first shown at table height for visual inspection. This plain letter B was constructed in such a way as to be symmetrical about a horizontal axis; that is, the top and bottom halves of the letter were the same size and shape. The subjects were told that, to familiarize themselves with the kind of forms that would be used, they could explore the letter tactually.

The normal and mirror judgments that subjects were required to make were demonstrated visually with the block letter while it remained horizontal, at table height: For the demonstration of the normal position, the top of the letter was the farthest part of the letter from the subject, and the curved sections of the B were to the subject's right. For the demonstration of the mirror position, the letter was repositioned, with the curved sections on the subject's left.

The subjects were then told that they were going to be presented the letters $\mathrm{F}$ and $\mathrm{P}$, one at a time, in various positions in the horizontal plane above their heads. The location of the upper board was shown. No attempt was made to show a letter in motion from the height of the table to that of the upper board. The subjects were told that they were to indicate, regardless of whether it was positioned upright or not, whether the letter was in the normal or the mirror position. They were then asked to label the normal and mirror positions of a letter shown visually at table height, using the letter $B$ that had already been presented to them in the instructions. Instruction continued until the subjects identified both positions (usually immediately).

Practice session. After being given their instructions, the subjects were asked to put on the blindfold. A B (on the mounting device) was positioned in the horizontal plane above the subject's head, so that the curved side was to their right. The subjects were not told whether this letter was in an upright or a not upright orientation. Nor were they asked. This was done to allow the subjects to come to their own assessment of these matters. The subjects were told to explore the letter, using both hands. They were free to con- 
Table 1

Mean Proportion of Normal Responses by Letter Orientation in Experiment 1

\begin{tabular}{|c|c|c|c|c|}
\hline \multirow[b]{3}{*}{ Front of Letter } & \multicolumn{4}{|c|}{ Top of Letter } \\
\hline & \multicolumn{2}{|c|}{ Near } & \multicolumn{2}{|c|}{ Far } \\
\hline & $M$ & $S D$ & $M$ & $\overline{S D}$ \\
\hline$\overline{\mathrm{Up}}$ & .03 & .08 & .93 & .17 \\
\hline Down & .63 & .39 & .01 & .05 \\
\hline
\end{tabular}

tinue practicing until they felt comfortable and were ready to begin the first test trial.

Test trials. In the test trials, the subjects reached up, above their heads, to the location of the letter affixed to the mounting device. A stiff, flat card placed under the letter was used to conceal the letter until the start of the trial. The subject's hands were guided into place under the card. The size and height of the apparatus forced the subjects to extend their arms upwards, to slightly above head level, while feeling the letter. Thus, letters were always felt palm up from underneath. When the card was removed, the subjects assessed the position of the letter by responding either "normal" or "mirror" out loud.

During the test stage, 16 trials were given to the subjects. Of these, half the presentations were with the letter F, and the other half with the letter $P$. The letters were presented in four positions (that is, each letter appeared twice in each orientation). The top of the letter was positioned either far from the subject (top:far) or near the subject (top:near). Also, the front of the letter was positioned so that it was facing either up or down (front:up and front:down, respectively). The two short horizontal bars of the $F$ and the loop of the $P$ are to the right of the vertical bars of the letters in the top:far/ front:up condition and in the top:near/front:down condition. Thus, varying the orientation of the top and front of the letter produced four possible orientations, each of which was presented twice.

The trials were divided into two blocks of eight, with each subject being exposed to each letter in each condition (two letters in four letter positions), once in each block. The four orientations were presented randomly per block. The normal/mirror assessment was recorded for each trial.

\section{Results and Discussion}

The mean proportions of "normal" responses for each orientation are shown in Table 1. A 2 (top:near or top:far) $\times 2$ (front:up or front:down) repeated measures analysis of variance (ANOVA) shows that the positions of the top $\left[F(1,24)=11.04, M S_{\mathrm{e}}=0.06, p<.005\right]$ and the front $\left[F(1,24)=9.57, M S_{\mathrm{e}}=0.05, p<.005\right]$ of the letters significantly affected subjects' assessments. Also, there is a significant top $\times$ front position interaction $[F(1,24)=$ $\left.317.58, M S_{\mathrm{e}}=0.05, p<.0001\right]$.

If the subjects are assessing orientation as if relying on positions reached via an elevator transformation, the top:far/front:up orientation should have the highest proportion of "normal" responses. Tukey's honestly significant difference (HSD) found this to be the case (HSD = $.30, p<.001)$, since this position was much higher (.93) than all the others. Also, if a secondary option favored by the subjects is a posture in keeping with the arc transformation, the top:near/front:down orientation should have a significantly higher proportion of "normal" responses (.63) than do the two remaining, lowest orientations (top: near/front:up, HSD $=.60, p<.001$, and top:far/front: down, HSD $=.63, p<.001)$. The remaining two options
(.03 and .01) are not significantly different $(\mathrm{HSD}=.02$, $p=.99)$ and are both infrequent.

Oldfield and Phillips (1983) reported that if subjects reached under a surface at table height to locate a small letter, they identified the shape as if the letter faced down, with its top far from the subject. In contrast, our subjects called the top:far/front:down position "normal" least often (.01). The letter position selected by the subjects in the Oldfield and Phillips study was the same as that shown by Figure 1C, if Figure 1 is held above one's head so that its captions can still be read. The Oldfield and Phillips study involved a continuous surface backing the letter. The letters were in a circular row on that surface. The subjects of Oldfield and Phillips may have treated the displays as if they were letters on a display surface, such as a page, with the page turned over to face away from them. They may have asked themselves what orientation the letter would be in if the page was turned back to face them and viewed from above (or if they moved in such a way as to face the "page" of letters). Our letters were suspended on columns or stems, with no continuous surface backing the letters as if on a page. We suggest that our subjects were unlikely to take individual letters that were physically above their head and ask what they would be like if the letters were turned over to reverse left for right and viewed from above the stands. If we are correct, Oldfield and Phillips were testing a situation related to turning pages, but our situation may offer evidence on how letters appear to be oriented in their current position and from the subject's present vantage point.

In sum, a strong preference is evident for a posture reached by an elevator transformation; a posture in keeping with an arc transformation is also used, but to a lesser extent.

\section{EXPERIMENT 2}

In this second experiment, several different letters were tested in the ceiling position through vision. In addition, two different fonts were used. One was plain, or sans serif. The other had serifs. We wished to demonstrate that the ceiling positions at which the top of the letter was nearest the subject were perceived as upright. We were not expecting any different effects from the font type of the letter, but we wanted to show that the uprightness judgment we described indeed occurs with both types of lettering. We provided letters in fonts that are rectangular and vertical in style, rather than italic or slanted, to ensure that questions of orientation would be unambiguous. The letters in plain sans serif font are ones we made ourselves from wood for the tactile experiments. So, in this experiment with vision, we wanted to demonstrate that similar effects occur with our simple lettering and also with a more ornate font type, commercially available, with evident fronts and backs, that would be more common in visual experience. We bought the ornate font letters from a hardware store. They are of a type used outside homes, shops, and so forth, where large letters, highly readable from a 
distance, are needed. We provided two letters in one font type and two in another. This experiment is by way of a demonstration of an effect that many would likely say is obvious, and certainly, a reader can check our account of the effects, using Figure 1.

\section{Method}

\section{Subjects}

Eight subjects $(5$ females, 3 males; mean age $=18.5$ years, range $=18-19$ ) participated in this experiment. All were volunteers from an undergraduate psychology course.

\section{Stimuli and Apparatus}

Two types of letters were used in test trials. One was relatively ornate with serifs, and the letters were uppercase letters $Y$ and $F$, made of wood. The backs of the letters were flat. The fronts had grooves, or routing, at their edges. The termini of the bars of the letters had transverse strokes (serifs) as decorative elements. A second kind of font was plain. The letters were uppercase letters $B$ and $J$, also made of wood. The bars had no terminal decorative strokes. The front was as flat as the back. Nothing distinguished the front of the letter from the back, except the identity of the letter. We will call these letters routed ( $\mathrm{Y}$ and $\mathrm{F})$ and plain $(\mathrm{B}$ and $\mathrm{J})$. The routed letters were approximately $24 \mathrm{~cm}$ high $\times 18 \mathrm{~cm}$ wide $\times 1.5 \mathrm{~cm}$ thick. The type of plain letters used in this experiment was also used in Experiments 1 and 3 with touch: The large letter size was chosen for easy identification and to increase the use of haptics (such as extensive hand motions). Finger presses can identify small letters - the finger pad detecting straight contours and curves. The letters were positioned above and slightly in front of the observer and were shown facing down, toward the observer.

The mounting device and all the other materials used were identical to those used in Experiment 1. The letters were shown face down.

\section{Procedure}

The subjects were tested individually. Each was asked to report whether a letter was upright or not. Letters were placed above head level in the horizontal plane. The letters were presented in two positions, relative to the observers: top:near and top:far. Each subject attempted four experimental trials, one each with Y, F, B, and J.

Instructions. A routed and plain block letter $\mathrm{P}$ (without an attached stand) was first shown flat on a table for visual inspection. The upright and not upright judgments subjects were required to make were demonstrated while the letters remained horizontal, at table height. For the demonstration of the upright position, the top of the letter was the furthest part of the letter from the subject, and the front of the letter was facing up toward the ceiling. For the demonstration of the not upright position, the letter was repositioned so that the top of the letter was near to the subject, while its front remained facing up. If Figure $I$ is placed horizontally on a table, the letters appeared like positions $A$ and $C$, respectively. They were then told that letters would be placed in various orientations above their heads in the horizontal plane. The location of the upper board was shown. They were then asked to label, with a letter at table height, upright and not upright positions, using one of the letter Ps already presented to them during the instructions. Instruction continued until the subjects identified both positions (usually immediately).

Test trials. In the test trials, the subjects looked up, above their heads, to the location of the letter affixed to the mounting device. Stiff, flat cards were used to conceal each letter until the start of the trial. When a card was removed, the subjects assessed the orientation of the letter by responding either "upright" or "not upright." During the test stage, four trials were given to subjects. Each of the four letters was presented once. One of the routed and plain letters was pre- sented so that its top was near to the subject (top:near) and its front was facing down. If Figure 1 is held above one's head so that its captions can still be read, the two downward facing orientations used were $A$ (top:near) and C (top:far). The other letters (one routed, one plain) were presented with the tops far from the subject (top:far) and the fronts facing down. Letters were assigned to top:near and top:far at random, with different orders for each subject.

\section{Results and Discussion}

A total score for each letter position (top:near or top:far) and each letter type (letter:routed or letter:plain) was obtained. The results were decisive. In all but one instance (a plain letter), the subjects assessed letters in the top:near position as being upright. Also, in all but one instance (a plain letter), the subjects described top:far positions as not upright. The exceptional instances came from the same subject.

The results of this experiment supported our expectations. Subjects deem positions in keeping with the arc transformation to be upright. We have reported this experiment with vision to make a point about visual judgments of uprightness using postures shown in Figures $1 \mathrm{~A}$ and $1 \mathrm{C}$. It may be worth noting that pilot studies with our wooden letters found what the reader will no doubt have observed by using Figures 1B and 1D held above his or her head. Placed above the head, wooden block letters such as F facing upwards away from the subject with the top of the letter near the subject and the bars on the left (Figure 1B) were judged to be upright but to be showing their backs to the subject. If the F had its top far from the subject but the bars on the right (Figure 1D), the letter was deemed to be inverted with its back to the subject.

\section{EXPERIMENT 3}

Our third experiment tests the perception of uprightness in touch. The procedure was similar to that of Experiment 1 . However, since uprightness can mean physically vertical as well as looking erect, which could influence the judgments we were asking for, we varied the initial position of the letter presented during instructions. That is, during the introduction, one group of subjects was shown a letter lying flat on a table. The subjects were told this position was an example of a letter being upright. The second group saw the letter standing on the table (standing vertical). These subjects were also told that this position was upright. We will call this difference the letterposition-during-instructions between-subjects variable. A letter flat on a table, as was the case in Experiment 1, might implicate an elevator transformation, whereas a letter set vertically might rule against an elevator transformation. Alternatively, the final location and posture of a letter being examined may be the key factors influencing its apparent orientation.

The results of Experiment 1 suggest that subjects should use positions reached by an elevator transformation for the most part, with ones in keeping with an arc a 
Table 2

Mean Proportion of Upright Responses by Group and Letter Orientation in Experiment 3

\begin{tabular}{|c|c|c|c|c|c|}
\hline \multirow[b]{3}{*}{ Instructions } & \multirow[b]{3}{*}{ Front of Letter } & \multicolumn{4}{|c|}{ Top of Letter } \\
\hline & & \multicolumn{2}{|c|}{ Near } & \multicolumn{2}{|c|}{ Far } \\
\hline & & $M$ & $S D$ & $M$ & $S D$ \\
\hline \multirow[t]{2}{*}{ Vertical } & Up & .22 & .25 & .91 & .13 \\
\hline & Down & .25 & .35 & .78 & .28 \\
\hline \multirow[t]{2}{*}{ Horizontal } & Up & .46 & .39 & .59 & .33 \\
\hline & Down & .53 & .45 & .50 & .44 \\
\hline \multirow[t]{2}{*}{ Overall } & $\mathrm{Up}$ & .34 & .34 & .75 & .29 \\
\hline & Down & .39 & .42 & .64 & .39 \\
\hline
\end{tabular}

notable but secondary preference. This means top:far positions should be reported as being upright more than are top:near positions, but top:near should be selected on occasion.

The direction in which a letter faces may have a relatively minor bearing on judgments of its uprightness. A person is upright even if he or she faces away from us. Similarly a page of letters is upright even if we view it through the back. Hence, we anticipated that the chief influence on judgments of uprightness should be the location of the top of the letter.

\[ \text { Method } \]
Subjects
Seventeen subjects (14 females, 3 males; mean age $=20.4$ years,
range $=19-30$ ) participated. One subject, who said he did not un-
derstand the instructions (male, age $=19$ ), was removed from the
analysis. All participated for credit in an introductory psychology
class.

\section{Stimuli and Apparatus}

The letters used in test trials were plain uppercase letters $J$ and $P$. All other materials were identical to those in Experiment 1.

\section{Procedure}

With the exception of the inclusion of the two uprightness instructions conditions, other procedures were identical to those of Experiment 1.

The subjects were randomly assigned ( 8 subjects each) to one of two letter-position-during-instructions conditions: vertical and horizontal. In the vertical condition, the visual inspection of upright and not upright positions with a practice letter $F$ was undertaken with the letter positioned perpendicular to the surface of the table. In this case, for the demonstration of upright, the bottom of the letter was on the surface of the table, with the front of the letter facing the subject. For the demonstration of the not-upright position, the top of the letter was resting on the table, and the front of the letter was still facing the subject. In the horizontal condition, the letter was positioned with its back resting on the table top. In this case, upright referred to the position in which the top of the letter was the part farthest from the subject. For the not-upright position, the top of the letter was nearest the subject.

\section{Results and Discussion}

\section{Instructions Condition}

The mean proportions of upright responses for each of the letter-positions-during-instructions conditions for the four letter positions were compared. We analyzed each in- structions condition separately, and we also analyzed the combined conditions.

An ANOVA on the vertical condition found top:near or top:far to be significant $\left[F(1,7)=49.09, M S_{\mathrm{e}}=0.06\right.$, $p<.001]$. However, the location of the front (front:up or front:down) was not significant $\left[F(1,7)<1, M S_{\mathrm{e}}=11\right]$, and there was no significant front $\times$ top interaction $\left[F(1,7)<1, M S_{\mathrm{e}}=0.05\right]$. There were no significant effects in the horizontal condition [top:near or top:far, $F(1,7)<$ $1, M S_{\mathrm{e}}=0.03$; front:up or front:down, $F(1,7)<1, M S_{\mathrm{e}}=$ 0.02 ; front $\times$ top, $\left.F(1,7)=1.84, M S_{\mathrm{e}}=0.03, p=.12\right]$.

The ANOVA on the combined results, shown in Table 2 , treated instructions (vertical or horizontal) as a betweensubjects variable and included two within-subjects variables (top:near or top:far; front:up or front:down). In this analysis, the difference between the two instruction groups was not significant $\left[F(1,14)<1, M S_{\mathrm{e}}=0.04\right]$. The instructions $\times$ top interaction $\left[F(1,14)=3.84, M S_{\mathrm{e}}=0.33\right.$, $p=.07]$ was not significant or was, at best, marginal. Also, the instructions $\times$ front $\left[F(1,14)<1, M S_{\mathrm{e}}=0.06\right]$ and the instructions $\times$ top $\times$ front $\left[F(1,14)<1, M S_{\mathrm{e}}=0.04\right]$ interactions were not significant. The analysis on the combined results only showed an effect for the position of the top of the letter $\left[F(1,14)=5.23, M S_{\mathrm{e}}=0.33, p<.05\right]$. The position of the front of the letter $\left[F(1,14)<1, M S_{\mathrm{e}}=\right.$ $0.06]$ and the top $\times$ front interaction $[F(1,14)=2.44$, $\left.M S_{\mathrm{e}}=0.04, p=.14\right]$ were not significant.

Tukey's HSD indicates that the two top:far positions (face:up and face:down) are not significantly different from one another $(\mathrm{HSD}=.11, p=.44)$. This is also the case for the two top:near positions. Evidently, the direction in which the letter faces (up or down) is immaterial. But both top:far positions are judged to be upright more than the two top:near positions (HSD $=.33, p>.05$ ).

The results are in line with those for Experiment 1. The subjects in Experiment 3 favored the posture reached by an elevator transformation, especially in the vertical instructions condition. The posture reached by an arc transformation was a secondary preference, rivaling the one in keeping with the elevator transformation in the horizontal condition. We speculate that results in the vertical condition may have been influenced by the fact that the top of letters set vertically are close to the position of the tops of the letters set in our top:far testing condition. But we stress that the top:near condition favored by vision did not win out, and both in the overall analysis and markedly in the vertical condition, the top:far position was deemed to be upright.

None of the results in Experiment 3 was close to $100 \%$ or $0 \%$ (which would indicate total agreement), unlike the case in the first experiment. This is due, we suggest, to the fact that letters are upright or inverted irrespective of the direction in which they face. In Experiment 1, the subjects were asked about a normal orientation, which implicates both correct left-right posture and uprightness. If the left-right posture of the letters in Experiment 1 was incorrect, the subject indicated that the orientation was not normal. We suggest that this would produce strong 
agreement that two positions were not normal. Only if the left-right positioning was correct did the upright criterion for normal become relevant in Experiment 1. As a result, in this account, in Experiment 1 the top:far position was accepted readily, and top:near was acceptable to a lesser extent.

In Experiment 3, the subjects were less likely to use left-right positioning to exclude a stimulus from consideration, but even so, the left-right positions of parts of the letter may have played a role in ameliorating the dominance of the position of the top of the letter. When the letter has its top far from the subject and is facing up, it has both its top and its left and right sides as they would be on a table in front of the subject. Thus, two factors could be said to favor this orientation (and the result is .75 in Experiment 3 for top:far/front:up). When it is top:near and front:up, it does not have either its top or its left and right sides as they would on a table (.34 in Experiment 3 ). The difference between the most-favored and least-favored condition is .41 . When it has its top far from the subject, as it would be on a table, and its left and right sides reversed, it could be said to have one factor in its favor, and it is indeed modestly acceptable (.64 in Experiment 3 ). When it has its top near to the subject - the reverse of its normal posture on a table-but its left and right sides are as they would be on a table, it again has one factor in its favor and reaches .39 in Table 3 . The difference between only the top being the factor in the letter's favor and only the left-right positioning being in its favor is only .26. It seems that both the location of the top and the left-right positioning of the sides may be factors in the subjects' judgments, although the location of the top is the more important.

\section{GENERAL DISCUSSION}

The present study examined the haptic perception of block letters placed above subjects' head level in the horizontal plane, studying whether assessments of apparent orientation through touch are similar to those that we argued are made through vision and that we asked the reader to test with Figure 1.

In Experiment 2, we substantiated our claim that, with vision, subjects favor positions reached via the arc transformation as being upright. That is, letter positions in which the top of the letter was near to them were assessed as being upright; positions where the top was far were not assessed as being upright. The judgments were almost unanimous.

Experiments 1 and 3, in which subjects used touch to assess the left-right positioning and uprightness of letters, respectively, indicate that subjects often assess the orientation reached via an elevator transformation as being upright and normal, although the orientation reached via an arc transformation is acceptable to some extent. Assessments of uprightness are generally dependent on the position of the top of the letter, and if the top is far from the subject, the letter is assessed as being upright.
Assessing that the letters are normal requires that the relative parts of the letter be distributed correctly to the subject's left and right, in addition to the letter being upright. It is impressive that judgments were close to unanimous in Experiment 1, with touch, when the letters were top:far and the left and right components were arranged as they are when the letter is at table height. Correspondingly, judgments were close to unanimous in Experiment 2 with vision, when the letters were top:near, and the left and right parts were as they would be at table height.

Why should touch favor different postures than vision? We suggest that haptic objects and their orientation in 3-D space are often assessed with the elevator transformation. Cups, drawers, and shelves will contain liquids, hold bulky items, or act as supports, respectively, if they are treated as if they should be raised vertically. On this account, characteristic haptic objects and tasks of manipulation may influence subjects using touch when assessing orientation, even when the object is one that is not generally treated in this fashion with vision.

Pushing further, we should ask why haptics might often be relatively labile, lending themselves to rivalrous criteria. Perhaps we can rephrase this question and gain some insight. Should there be a variety of apparent vantage points? Would that be in the very nature of haptics? Well, surely yes. Haptics operates across time. The hands are mobile. We explore particular objects or arrangements of objects from many directions in three dimensions. We can contact an object's front, top, back, and sides when we explore it tactually. The direction of the contacts changes swiftly. Two directions can be used simultaneously. Interestingly, we could define the perceiver's vantage point as the place around which objects lie in various directions. If haptics is accustomed to varying the direction from which we contact the object, this should allow a good deal of variability in the use of vantage points in tactile tasks. When we vary those directions, we plan possible motions from a vantage point (for instance, from above or below an object). We take into consideration motions not yet undertaken and some of the effects of changing our vantage point (for instance, from above or below). If so, haptics is labile for good reasons. It requires us, at times, to consider many vantage points and directions in 3-D space around us.

This analysis of lability can be used to rethink the distinctions we have used here from the start. Indeed, our geometrical description of the movements of Figure 1 in our introductory section, contrasting arcs and elevators, can be recast to contrast two vantage points, one occupied physically by the observer and one imaginary or apparent. One arc transformation is around the observer's head as a fixed vantage point (Figure 1A moving in an arc before our eyes), and the other could be around an apparent vantage point far above the ceiling, sufficiently far that the elevator motion we described would not be material. If the apparent vantage point is placed far enough above the ceiling, large motions of a display in front of an observer would have no effect on its apparent orientation. Is this 
really what subjects entertain in processing the stimuli? We suspect not for most subjects (although we could, of course, instruct subjects to analyze the stimuli in this fashion). However, it is instructive that a lowercase b drawn on the subject's forehead, its bulge to the subject's right, and $a b$ drawn on the back of the subject's head, bulge likewise toward the subject's right, are both judged to be $b$. The latter judgment especially suggests flexibility in the subject's vantage point and that of a disembodied eye (Concoran, 1977) located some way behind the subject's head. That imaginary outside-the-head vantage points are relevant to touch is not as preposterous as it may seem at first blush.

We admit that the psychological reality of one or the other description remains as an intriguing problem for research. It is also a daunting problem. It is intrinsic to any spatial puzzle that many roads lead to Rome. An infinite number of different combinations of rotations in the plane, left-right changes, and motions in an arc or vertically can produce the same results physically. Correspondingly, subjects may combine transformations in different orders when making spatial judgments. Also, we anticipate that the observer may be influenced by some changes, especially left-right ones, to consider changing his or her apparent vantage point or moving the stimulus to a new apparent position. Kennedy (1993) describes the subject's paraphrasing spatial changes when inventing different ways to Rome. Recognizing this element of flexibility in internal processing of routes between two physical endstates, in our analysis, we have chosen to emphasize the physical geometry of the stimuli we presented. But we are not just "black box" theorists. We acknowledge the need to dovetail our analyses with considerations of psychological processes-notably, ones supporting the observer's ability to adopt a wide variety of apparent vantage points.

In conclusion, we suggest that a lesson here about haptic's dealings with letters and orientation is that the results in some conditions run counter to those with vision. Criteria in keeping with an arc transformation around the observer's head are dominant with vision, but ones conforming to an elevator rise are evident with touch and, at times, dominate in the subject's responses. We also note that there is lability in judgment in the tactile tasks, and we suggest that this fits with the fact that objects are approached from many directions in haptics.

\section{REFERENCES}

Concoran, D. J. W. (1977). The phenomenon of the disembodied eye or is it a matter of personal geography? Perception, 6, 247-253.

DAY, R. H. (1990). The Bourdon illusion in haptic space. Perception \& Psychophysics, 47, 400-404.

Garbin, C. P. (1988). Visual-haptic perceptual nonequivalence for shape information and its impact upon cross-modal performance. Journal of Experimental Psychology: Human Perception \& Performance, 14, 547-553.

Garbin, C. P., \& Bernstein, I. H. (1984). Visual and haptic perception of three-dimensional solid forms. Perception \& Psychophysics, 36, 104-110.

GIBson, J. J. (1979). The ecological approach to visual perception. Boston: Houghton \& Mifflin.

JONES, B. (1981). The developmental significance of cross modal matching. In R. D. Walk \& H. L. Pick, Jr. (Eds.), Intersensory perception and sensory integration (pp. 71-108). New York: Plenum.

KenNedy, J. M. (1993). Drawing and the blind: Pictures to touch. New York: Yale University Press.

KENNEDY, J. M. (1997, January). How the blind draw. Scientific American, 276, 76-81.

Kennedy, J. M., Gabias, P., \& Heller, M. A. (1992). Space, haptics and the blind. Geoforum, 23, 175-189.

MARKs, L. E. (1978). The unity of the senses: Interrelations among the modalities. New York: Academic Press.

MARMOR, G. S., \& ZABACK, L. A. (1976). Mental rotation by the blind: Does mental rotation depend on visual imagery? Journal of Experimental Psychology: Human Perception \& Performance, 2, 515-521.

NATSOULAS, T. (1966). Locus and orientation of the perceiver (ego) under variable, constant, and no perspective instructions. Journal of Personality \& Social Psychology, 3, 190-196.

OLDFIELD, S. R., \& PHILliPS, J. R. (1983). The spatial characteristics of tactile form perception. Perception, 12, 615-626.

RUdel, R. G., \& TEUBER, H.-L. (1964). Decrement of visual and haptic Mueller-Lyer illusion on repeated trials: A study of crossmodal transfer. Quarterly Journal of Experimental Psychology, 15, 125-131.

SeKIYAMA, K. (1991). Importance of head axes in perception of cutaneous patterns drawn on vertical body surfaces. Perception \& Psychophysics, 49, 481-492.

Shimojo, S., SASAKI, M., Parsons, L. M., \& ToriI, S. (1989). Mirror reversal by blind subjects in cutaneous perception and motor production of letters and numbers. Perception \& Psychophysics, 45, 145-152.

(Manuscript received February 11, 1997; revision accepted for publication May 29, 1998.) 